\title{
ORIGINAL
}

\section{ANÁLISIS DE LAS PRINCIPALES VARIABLES DETERMINANTES DE LA EVOLUCIÓN DE LA MORTALIDAD POR DEMENCIAS EN ANDALUCÍA}

\author{
Miguel Ruiz Ramos
}

Servicio de Información y Evaluación. Consejería de Salud de La Junta de Andalucía.

\section{RESUMEN}

Fundamentos: Las demencias son una de las causas de mortalidad que más han aumentando en los último años en los países desarrollados. El objetivo de este trabajo es analizar la mortalidad por edad, sexo, estado civil, así como su evolución temporal en Andalucía en el periodo de 1999 al 2010.

Métodos: Con las defunciones por demencias de los mayores de 59 años del Instituto de Estadística de Andalucía y las poblaciones correspondientes, se calcularon las tasas de mortalidad bruta, estandarizadas por edad y sexo, y específicas por edad; mediante regresión joinpoint se calculó los porcentajes anuales de cambio; y además con las estimaciones de las poblaciones por estado civil y sus defunciones calcularon las tasas brutas y estandarizadas por edad, sexo y estado civil.

Resultados: Las tasas de mortalidad estandarizadas pasaron de 124,8 a 161,0 defunciones por 100.000 en las mujeres y de 110,3 a 147,7 en los hombres, el incremento anual fue del $4,2 \%$ y $3,8 \%$ en mujeres y hombres. Las mujeres murieron más que los hombres con una razón de tasas estandarizadas entre 1,08 y 1,29 . Y, la edad fue la variable que más determinó la mortalidad.

Conclusiones: La mortalidad por demencias en Andalucía ha experimentado un importante incremento en los últimos doce años y continuará aumentando, con el consiguiente impacto sanitario y social que tienen este grupo de enfermedades configurándose como un importante problema de salud.

Palabras clave: Andalucía. Demencias. Epidemiología. Mortalidad Tendencias.

\section{ABSTRACT \\ Analysis of Variables Related with Dementia Mortality Trend. Andalusia, Spain}

Background: The dementias are a cause of mortality have increased over the last years. Therefore is important to analyze the variables more related to its development in Andalusia between 1999 and 2010.

Methods: With the deaths of 60 and over by dementia from Andalusia Statistical Institute and the populations from corresponding years, are estimated crude mortality rates, standardized and age-specific; by joinpoint regression was calculated percentages annual change; and also, with the population estimates by marital status and deaths was calculated crude rates and standardized for age, sex and marital status.

Results: The standardized mortality rates increased from 124.8 to 161.0 deaths per 100,000 in women and 110.3 to 147.7 in men, the annual increase was $4.2 \%$ and $3.8 \%$ in women and men. The women died more than men with a standardized rate ratio between 1.08 and 1.29. Age was the variable that determined mortality.

Conclusions: Mortality from dementia in Andalusia has increased over the past 12 years and will continue to increase with the consequent social and health impacts posed by these diseases, configured as a major health problem.

Key words: Andalusia. Dementia. Epidemioligy. Mortality. Trends.

Correspondencia

Miguel Ruiz Ramos

Consejería de Salud de la Junta de Andalucía

Avenida de la Innovación s/n. Ed. Arenas 1.

41020 Sevilla

miguel.ruiz.ramos@juntadeandalucia.es 


\section{INTRODUCCIÓN}

La demencia es un síndrome adquirido y persistente de deterioro de varias funciones mentales superiores, producido por patología orgánica en una persona sin alteraciones del nivel de conciencia, y que le produce una incapacidad en la actividad social y laboral. Uno de los mayores avances en su estudio ha sido la identificación de diferentes tipos clínicos, producidos por distintos procesos patológicos ${ }^{1}$. Han pasado de ser consideradas problemas mentales que se debían resolver en el ámbito sociosanitario a ser enfermedades con posibilidad de tratamiento debido a las nuevas evidencias en cuanto a sus causas y, sobre todo, al aumento de la prevalencia, constituyendo en los países desarrollados un problema importante en el ámbito tanto clínico como de salud pública. En este sentido la Organización Mundial de la Salud puso de manifiesto su importancia, mostrando como las demencias son las principales responsables de los Años de Vividos con Discapacidad $^{2}$.

Dado el crecimiento sostenido de la población mayor de 65 años, se espera que el número de personas afectadas por estas patologías aumente progresivamente. Se ha estimado que en los últimos años la prevalencia ha alcanzado el 3,9\% de la población mundial, proyectándose un incremento al 6,9\% para el año 2020 y al $13,1 \%$ para el año $2040^{3,4}$.

La investigación epidemiológica de las demencias se ha realizado con estudios transversales y de cohortes que han permitido determinar las tasas de prevalencia, de incidencia y de mortalidad ${ }^{5,6}$. Desde hace unos años se están creando registros de demencias que en un futuro suministraran información muy valiosa sobre estas enfermedades en España ${ }^{7,8}$. Pero desde la perspectiva poblacional existen pocos indicadores para conocer la prevalencia y tendencia de este importante problema de salud pública y aunque la mortalidad no es un buen indicador para conocer qué ocurre en la población con las enfermedades crónicas degenerativas que son altamente prevalentes pero producen pocas defunciones, en el caso de las demencias, por su origen orgánico $\mathrm{y}$ el deterioro físico que producen, pueden ser consideradas causas importantes de defunción ${ }^{9}$. Las demencias irreversibles en las que se incluye la enfermedad de Alzheimer, fueron la octava causa de mortalidad en 2003 y la séptima en los años del 2004 al 2006 en los Estados Unidos $^{10,11}$. En España el 11 por ciento de todas las muertes están relacionadas con las demencias y alcanzan hasta un tercio de las muertes de las personas de más de 85 años $^{12}$. Las demencias pueden considerarse causas significativas de defunción y por lo tanto los indicadores de mortalidad pueden ser útiles para su seguimiento en el conjunto de la población.

El objetivo del presente trabajo es describir las variables que determinan la evolución de la mortalidad por demencias en la población de sesenta años y más en Andalucía.

\section{MATERIAL Y MÉTODOS}

Se utilizaron las defunciones por demencias de los años 1999 al 2010 de personas de 60 y más años de edad residentes en Andalucía, procedentes del Registro de Mortalidad de Andalucía. Se incluyeron todas las defunciones en las que la causa básica de muerte (aquella que origina el proceso letal) fuera una demencia. Las causas seleccionadas, para todo el periodo, fueron codificadas con la Clasificación Internacional de Enfermedades $10^{\mathrm{a}}$ revisión (CIE-10), y se correspondieron con los códigos F01: 
demencias vasculares, F03: demencias no especificadas y G30: enfermedad de Alzheimer. Además del lugar de residencia, la edad, y el sexo se estudió el estado civil de los fallecidos.

Las poblaciones necesarias para calcular indicadores procedieron de los datos oficiales de los padrones elaborados por el Instituto Nacional de Estadística (INE).

Se calcularon las tasas de mortalidad estandarizadas por edad y sexo mediante el método directo, utilizando como referencia la población estándar europea, y las tasas específicas por grupos de edad para los periodos de 1999-2004 y 20052010 , con las que se construyeron las curvas de mortalidad por edad. El análisis se estratificó por sexo.

La tendencia de la tasa estandarizada (Ts) por sexo y edad fue analizada mediante "joinpoint regression"13. Se utilizó como variable dependiente la Ts y como independiente el año de defunción. El método permitió detectar los segmentos que mejor describen los datos. El año en que se produjo el cambio se detectó en la serie de años estudiados y se denominó "joinpoint" y los joinpoints significativos se conservaron en el modelo final. El máximo número de joinpoints permitido para cada periodo fueron tres. El porcentaje anual de cambio esperado (PAC) se calculó al describir la tendencia del periodo.

Con las poblaciones por estado civil de los hombres y mujeres por grupos quinquenales de edad del censo del 2001 se calcularon los porcentajes que correspondían a solteros/a, casado/a, viudo/a y separado/a, y con esto se calcularon los correspondientes poblaciones por estado civil del periodo de 2005 al 2009, que era el último periodo de los que se disponía de datos definitivos de estas variables. Con estos datos se estimaron las tasas brutas y estandarizadas por edad para cada uno de los estados civiles

\section{RESULTADOS}

En Andalucía entre 1999 y 2010 se declararon 31.710 defunciones por demencias en la población de personas mayores de 59 años, lo que supuso el $4,8 \%$ del todas las muertes de residentes en Andalucía de esa edad. En la tabla 1 se presentan los principales indicadores que reflejaron la evolución de las demencias en la población. Mientras que el aumento de la población mayor de 59 años entre 1999 y 2010 creció el 19\% en las mujeres y el $22 \%$ en los hombres, el número de muertes por demencias lo hizo en $85 \%$ y $97 \%$, pasando de 1.905 defunciones en 1999 a 3.604 en el 2010.

Cuando se relacionaron las defunciones con el tamaño de la población mediante las tasas de mortalidad brutas se observó un importante incremento, pasando de 164,7 defunciones por 100.000 mujeres en 1999 a 255,7 por 100.000 en 2010 y en los hombres pasó de 104,1 por 100.000 a 167,9 por 100.000 entre los mismos años. Cuando se ajustaron por edad las tasas para poder comparar la mortalidad se mantuvo el mismo incremento (figura 1). El análisis de las tendencias mostró cómo las mujeres tuvieron un PCA del 3,8\% y los hombres del 3,5\% sin ningún punto de inflexión en todos los años de la series, siendo el aumento estadísticamente significativo tanto en las mujeres como en los hombres (figura 1).

La razón de tasas estandarizas entre mujeres y hombres mostró una mayor mortalidad por demencias en las primeras una vez controlado el efecto de la edad mediante el ajuste. La sobremortali- 
Tabla 1

Población, defunciones, tasas brutas, ajustadas y teóricas de mortalidad por demencias, y razón de tasas estandarizada mujeres/hombres Andalucía de 1999 a 2010

\begin{tabular}{|c|c|c|c|c|c|c|c|c|c|}
\hline \multirow{2}{*}{$\begin{array}{c}\text { Año de } \\
\text { muerte }\end{array}$} & \multicolumn{5}{|c|}{ Mujeres } & \multicolumn{5}{c|}{ Hombres } & \multirow{2}{*}{$\begin{array}{c}\text { Razón } \\
\text { de Ts }\end{array}$} \\
\cline { 2 - 10 } & Población & Def & Tb & Ts & Población & Def & Tb & Ts & \\
\hline 1999 & 77.7998 & 1281 & 164,7 & 124,8 & 599.344 & 624 & 104,1 & 110,3 & 1,13 \\
\hline 2000 & 784.910 & 1270 & 162,4 & 119,4 & 601.112 & 650 & 108,1 & 110,4 & 1,08 \\
\hline 2001 & 800.268 & 1398 & 174,7 & 125,4 & 617.298 & 609 & 98,7 & 97,3 & 1,29 \\
\hline 2002 & 803.302 & 1445 & 179,9 & 127,1 & 620.549 & 677 & 109,1 & 108 & 1,18 \\
\hline 2003 & 813.316 & 1663 & 204,5 & 143 & 629.578 & 787 & 125 & 122 & 1,17 \\
\hline 2004 & 826.078 & 1548 & 187,4 & 130,5 & 641.761 & 802 & 125 & 120,5 & 1,08 \\
\hline 2005 & 843.004 & 1952 & 231,6 & 158,8 & 657.468 & 868 & 132 & 124,6 & 1,27 \\
\hline 2006 & 86.275 & 1888 & 219,5 & 148,2 & 674.198 & 968 & 143,6 & 134,1 & 1,11 \\
\hline 2007 & 872.005 & 2050 & 235,1 & 157,5 & 686.096 & 1007 & 146,8 & 135,9 & 1,16 \\
\hline 2008 & 888.957 & 2223 & 250,1 & 163,5 & 702.542 & 1025 & 145,9 & 130,5 & 1,25 \\
\hline 2009 & 910.720 & 2224 & 244,2 & 157,9 & 718.629 & 1147 & 159,6 & 141,1 & 1,12 \\
\hline 2010 & 927.786 & 2372 & 255,7 & 161 & 733.771 & 1232 & 167,9 & 147,7 & 1,09 \\
\hline
\end{tabular}

Def: defunciones; Tb: tasa bruta; Ts: tasa estandarizad; razón mujeres/hombres; las tasas son por $10^{5}$ personas

dad femenina ocurrió en todos los años de la serie con importantes fluctuaciones entre ellos (tabla 1).

En la figura 2 se presentan las tasas de mortalidad específicas por edad. En mujeres y hombres se incrementó la mortalidad a medida que aumentó la edad, siendo más acusado a partir del grupo de edad 70-74 años. Destacó el incremento en las mujeres durante el periodo de 2005 a 2010 en particular en los grupos extremos de edad. Los hombres tuvieron menor mortalidad en todos los grupos de edad, de tal manera que las tasas del periodo 2005-2010 para los hombres se superpuso a las de las mujeres del 19992004.
En la tabla 2 se presentan los resultados de la mortalidad por demencias y estado civil para el periodo del 2005 al 2009. En los hombres eran los viudos los que mayores tasas brutas de mortalidad tuvieron, pero cuando se ajustó por edad fueron los solteros con una tasa de 230,2 por 100.000 solteros los que presentaron mayor mortalidad, seguidos de los viudos y de los casados y en último lugar los divorciados. En las mujeres las tasas brutas seguían el mismo orden que en los hombres pero volvieron a ser las solteras las que mayor Ts de mortalidad presentaron con 217,2 defunciones por 100.000 mujeres solteras, seguidas por las viudas, y divorciadas y casadas con muy poca diferencias entre estas dos últimas. 
Figura 1

Tendencia de la mortalidad por demencias en Andalucía de 1999 a 2010,

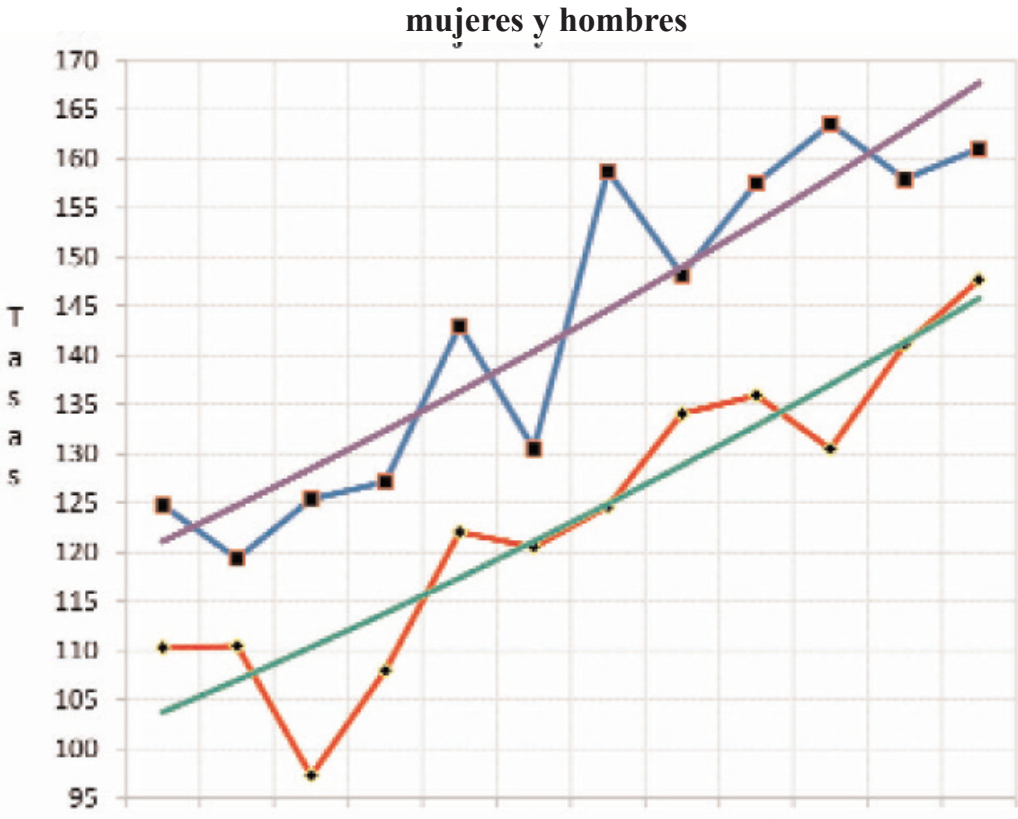

$199920003200120002 \quad 200032004200520062007200820092010$

Año defunción

-Mujeres $=$ MujereslainPoint $=$ Hombres - HombresJaintFoint

Figura 2

Tasas de mortalidad específicas por edad en los periodos de 1999-2004 y 2005-2010 para mujeres y hombres en Andalucía, 1999-2010

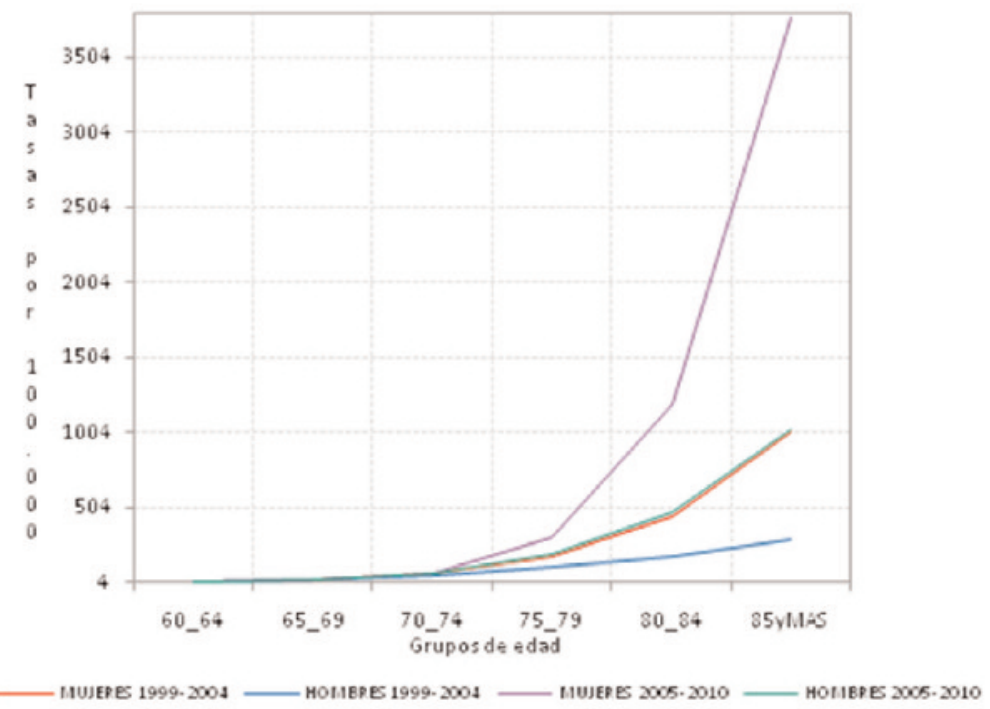


Tabla 2

Mortalidad por demencias y estado civil en Andalucía en el periodo de 2005 al 2009

\begin{tabular}{|l|c|c|c|c|}
\hline Sexo/indicadores & Soltero/ra & Casado/a & Viudo/a & Divorciado/a \\
\hline Hombres \\
\hline Defunciones & 383 & 3159 & 1438 & 38 \\
\hline $\mathrm{Tb}$ & 197,1 & 112,3 & 393,6 & 58 \\
\hline $\mathrm{Ts}$ & 230,2 & 121,1 & 152,1 & 93,5 \\
\hline Mujeres & \multicolumn{5}{|l|}{} \\
\hline Defunciones & 1130 & 1845 & 7316 & 45 \\
\hline $\mathrm{Tb}$ & 392,9 & 82,7 & 408 & 70,6 \\
\hline $\mathrm{Ts}$ & 217,2 & 139,5 & 158,6 & 142,3 \\
\hline
\end{tabular}

Figura 3

Mortalidad por demencias y estado civil en Andalucía en el perido de 2005 al 2009

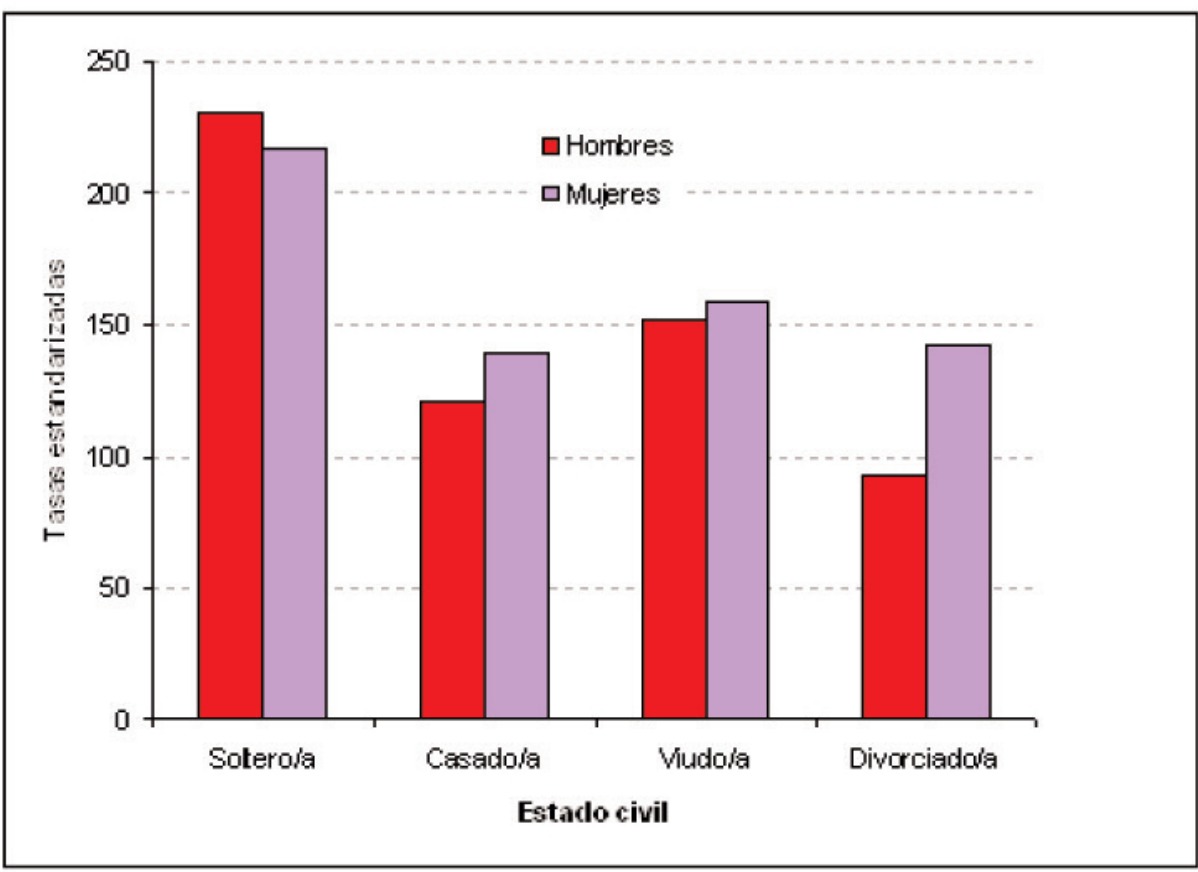


Cuando se comparan las tasas de hombres y mujeres (ver figura 3), sólo los solteros tenían mayor mortalidad que las solteras en los demás estados civiles eran las mujeres.

\section{DISCUSIÓN}

Durante la última década, la mortalidad por demencias en Andalucía se ha incrementado, concentrándose en los grupos de edad de personas más mayores. Las mujeres tienen mayor mortalidad que los hombres una vez controlados los efectos de la edad, lo que supone una característica distintiva de las demencias, ya que prácticamente en el resto de causas producen una sobremortalidad masculina.

Como ocurre en el resto de países desarrollados, parece claro que el envejecimiento de la población andaluza en los próximos años aumentará el número de personas que morirán por demencias $\mathrm{y}$, por lo tanto, es de suponer que también aumentará el número de personas que vivirá con ellas. Los datos de mortalidad por demencias suministran esta valiosa información y muestran que en Andalucía el número de personas fallecidas por estas causas se ha duplicado prácticamente en el periodo estudiado, pero los resultados ponen de manifiesto que aún manteniendo una misma estructura de edad de la población andaluza mediante la estandarización, también se ha producido un incremento importante. Todo ello indica que las demencias están aumentando independientemente del envejecimiento de la población, aunque el aumento de la edad media de la población andaluza ha contribuido y continuará haciéndolo, al ser la edad la variable más relacionada con el riesgo de morir por demencias, aunque hay que seguir buscando otras causas que expliquen el aumento.

En este sentido, como se dijo en la introducción, las demencias son un grupo de enfermedades que, independientemente de la etiología, comparten una clínica común. En este trabajo se han agrupado las demencias vasculares junto con la enfermedad de Alzheimer, repartiéndose las defunciones al cincuenta por ciento. No es el objetivo entrar en los aspectos clínicos patológicos de este grupo de enfermedades, pero no hay que dejar de mencionar las evidencias existentes en cuanto a las mejoras en diagnóstico y tratamiento ${ }^{14}$ que posiblemente estén influyendo en una mejor declaración como causas de muerte, aunque por lo publicado en otros países todavía exista una subnotificación de las demencias como causas de defunción ${ }^{15,16}$.

Así, se estima que sólo la mitad de los pacientes con demencias son diagnosticados por los servicios sanitarios. En Estados Unidos ${ }^{17}$ se ha comparado la incidencia de demencias basada en los casos declarados por los servicios sanitarios y las obtenidas por seguimiento de pacientes, resultando falta de coincidencia cuando se trata de demencias moderadas, lo cual mejora considerablemente cuando aumentan la gravedad de la enfermedad. Los médicos que hacen el diagnóstico de las demencias deben considerar que éstas pueden tener un papel importante como causas de muerte, pero no es fácil hacer un buen diagnóstico cuando la muerte ocurre en un contexto de pluripatología, como en el caso de las personas mayores. Por lo tanto, aun con las mejoras diagnósticas, en los certificados de defunción se subestiman las muertes por esta patología Esta situación se ha descrito en el Reino Unido $^{18}$ y Estados Unidos ${ }^{19}$, además algunos autores han demostrado que las demencias no fueron mencionadas en el certificado de defunción aunque el diagnóstico estuviese correctamente hecho ${ }^{20}$. Es probable que los certificados de defunción reflejen la prevalencia de las demencias avanzadas y de todas maneras suministran una información valiosa sobre este 
grupo de enfermedades ${ }^{21}$. Es decir, las demencias que alcanzan una estadio clínico terminal suministran características demográficas de las personas con estas patologías que son recogidas por los certificados de defunción realizados en el momento de la muerte, como se ha puesto de manifiesto en un trabajo realizado en Francia recientemente ${ }^{22}$.

La mayor mortalidad de las personas con demencias respecto a la población general ha sido documentada ampliamente $\mathrm{e}^{23-26}$ y los estudios derivados de los registros de demencias pueden suministrar información en este sentido. Sin embargo, estudiar la mortalidad por demencias con datos procedentes de registros poblacionales, como los procedentes de las estadísticas oficiales de causa de muerte, es menos frecuente. Se han publicado resultados similares a los de este trabajo en un estudio realizado en Cataluña ${ }^{27}$, aunque la magnitud de las tasas y los periodos temporales sean diferentes. En Estados Unidos la mortalidad atribuida a la enfermedad de Alzheimer experimentó un gran aumento en la décadas de los años ochenta y noventa y los autores lo atribuyen, al menos en parte, a una mejora diagnóstica ${ }^{19}$.

En el mundo se han realizado muchos estudios sobre prevalencia de las demencias y, con las variaciones debidas a las diferentes metodologías utilizadas ${ }^{28}$, han puesto de manifiesto una fuerte asociación entre la edad y la incidencia y prevalencia de las demencias ${ }^{29}$, llegando a plantear si está relación entre edad y demencia podría ser inevitable y por tanto llegar a afectar a todas las personas que mueren muy mayores, al final de sus vidas las personas longevas terminarían con algún tipo de demencia, sin embargo en este metaanálisis se concluye que la prevalencia de demencias en las personas que viven más de noventa años disminuye con respecto a grupos de edad de personas menores, suponiendo un resultado importante por sus implicaciones en salud pública y asistencia social de este grupo de población cada vez más numeroso.

La relación entre sexo y demencias en los estudios de prevalencia ha sido inconsistente, en muchos de ellos las mujeres presentaban mayores tasas que los hombres. Existen, sin embargo, diferencias estadísticamente significativas entre sexos en los grupos de edad muy mayores, en los que hay pocos hombres $\mathrm{y}$, por lo tanto, pocas demencias, aunque a este respecto las estimaciones son poco cla$\operatorname{ras}^{30,31}$. Por otra parte, los estudios de población con demencia demuestran una sobremortalidad masculina ${ }^{32}$, de manera que las mujeres con demencia sobreviven hasta edades más avanzadas que los hombres, con lo que acumulan una mayor proporción de casos $\mathrm{y}$, por lo tanto, la prevalencia femenina es más alta ${ }^{33}$.

La relación entre demencias y estado civil se ha estudiando en numerosos trabajos de prevalencia que muestran un mayor riesgo de las personas solteras en comparación con otros estados civiles ${ }^{34}$. En los resultados de este trabajo una vez ajustado el indicador por edad, las mayores tasas de morir por demencias las presentaron también los solteros. Este resultado habrá que analizarlo con mayor grado de detalle en próximos estudios, partiendo de la idea de que el estado civil declarado en las estadísticas oficiales no recoge con exactitud la situación real de las personas, aunque en los mayores de 60 años posiblemente la valoración de esta variable sea diferente que a edades mas jóvenes.

El ajuste de las tasas por edad mediante los modelos de regresión joinpont permite realizar un ejercicio de previsión de cuál será la magnitud de la mortalidad por demencias en los próximos años. Puesto que la mortalidad por esta causa depende en gran medida de su gravedad, que lógi- 
camente afecta de forma más importante cuanto más se acercan a la fecha de la muerte y es durante este periodo cuando mayores recursos sanitarios se consu$\operatorname{men}^{35}$, los resultados de este trabajo pueden ser útiles para conocer el futuro de este problema de salud y actuar en consecuencia.

Con la limitaciones expresadas más arriba sobre la calidad de los diagnósticos de demencias recogidos en los certificados de defunción y las derivadas de los métodos utilizados para medirlas, se puede concluir que la mortalidad por demencias son un importante problema de salud pública que habrá que seguir analizando en futuras investigaciones con datos que complementen a los de mortalidad y en los que se incluyan tanto variables de relevancia clínica como sociales.

\section{BIBLIOGRAFÍA}

1. Slachevsky \& Oyarzo. Las demencias: historia, clasificación y aproximación clínica. En: Tratado de Neuropsicología Clínica. Labos E, Slachevsky A, Fuentes P, Manes E editores. Buenos Aires: Librería Akadia 2008.

2. Murray CJ, Lopez AD, Jamison DT. The global burden of disease in 1990: summary results, sensitivity analysis and future directions. Bull WHO. 1994; 72: 495-509.

3. Brookmeyer R, Gray S. Methods for projecting the incidence and prevalence of chronic diseases in aging populations: application to Alzheimer's disease. Stat Med. 2000; 19: 1481-93.

4. Ferri CP, Prince M, Brayne C, Brodaty H, Fratiglioni L, Ganguli $\mathrm{M}$ et al.: Global prevalenceof dementia: a Delphi consensus study. Lancet. 2005; 366(9503): 2112-7.

5. Van der Flier WM, Scheltens P. Epidemiology and risk factors of dementia. J Neurol Neurosurg Psychiatry. 2005; 76 (Suppl): S2-7.

6. Del Barrio JL, De Pedro-Cuesta J, Boix R, Acosta J, Bergareche A, Bermejo-Pareja F, et al. Dementia, stroke and Parkinson's disease in Spanish populations: a review of door-to-door prevalence surveys. Neuroepidemiology. 2005; 24: 179-88.
7. López-Pousa S, Garre-Olmo J, Monserrat-Vila S, Boada M, Tárraga LL, Aguilar M, et al. Propuesta para un registro clínico de demencias. Rev Neurol. 2006; 43: 32-8.

8. Garre-Olmo J, López-Pousa S, Monserrat-Vila S, Pericot-Nierga I, Turon-Estrada A, Lax-Pericall C. Viabilidad de un registro demencias: características clínicas y cobertura diagnóstica. Rev Neurol. 2007 44(7):385-91.

9. Kelman HR, Thomas C, Kennedy GJ, Cheng J. Cognitive impairment and mortality in older community residents. Am J Public Health. 1994; 84: 125560 .

10. Heron, M. Deaths: Leading causes for 2004. National vital statistics reports. 2007. Disponible en: http://www.cdc.gov/nchs/data/nvsr56/nvsr56_05.pdf

11. Heron M, Hoyert DL, Murphy SL, Xu J, Kochanek KD, Tejada-Vera, B. Division of Vital Statistics. Deaths: Final data for 2006. National vital statistics reports. 2009. [citado el 23/11/2011] Disponible en: http://www.cdc.gov./NCHS/data/nvsr/nvsr57/nvsr57 _14.pdf.

12. Villarejo A, Benito-León J, Trincado R, Posada IJ, Puertas-Martín V, Boix R, et al. Dementia-Associated Mortality at Thirteen Years in the NEDICES Cohort Study. J Alzheimers Dis. 2011; Jun 21 MID: 21694455 [PubMed - as supplied by publisher)

13. Kim HJ, Fay MP, Feuer EJ, Midthune DN: Permutation tests for joinpoint regression with application to cancer rates. Stat Med. 2000; 19:335-51. Correction: 2001, 20: 655.

14. Hoyert DL, Rosenberg HM. Mortality from Alzheimer's disease: An update. Natl Vital Stat Rep. 1999;47(20):1-8.

15. Wachterman M, Kiely DK, Mitchell Sl. Reportin demential on death certificates of nursing home residents dying with end-stage dementia. JAMA. 2008; 300: $2608-10$

16. Olichney JM, Hofstetter CR, Galasko D, Tha LJ, Katzman R. Death certificate reporting of dementia and mortality in an Alzheimer's disease research center cohort. J Am Geriatr Soc. 1995; 43(8):890-893.

17. Tyas SL, Tate RB, Wooldrage K, Manfreda J, Strain LA. Estimating the incidence of dementia: the impact of adjusting for subject attrition using health care utilization data. Ann Epidemiol. 2006 ;16(6):477-84. 
18. Griffiths C, Rooney C, Trends in mortality from Alzheimer's disease, Parkinson's disease and dementia England and Wales, 1979-2004 Health Stat Q. $2006 ; 30: 6-14$.

19. Hoyert DL, Rosenberg HM. Alzheimer's disease as a cause of death in the United States Public Health Rep. 1997 ; 112 : 497-505

20. Ostbye T, Steenhuis R, Wolfson C, Walton R, Hill G. Mortality in elderly Canadians with and without dementia: a 5-year follow-up. Neurology. 1999 ; 53 : 521-526.

21. Raiford K, Anton-Johnson S, Haycox Z, Nolan K, Schaffer A, Caimano C, et al. CERAD part VII: accuracy of reporting dementia on death certificates of patients with Alzheimer's disease Neurology. 1994; 44 : 2208-2209

22. Brosselin P, Duport N, Bloch J. Mortality with Alzheimer's disease and dementia in France, 2006. Rev Epidemiol Sante Publique. 2010; 58(4):269-76.

23. Agüero-Torres H, Fratiglioni L, Guo Z, Viitanen M, Winblad B. Mortality form dementia in advanced age: A 5-year follow-up study of incident dementia cases. J Clin Epidemiol. 1999;52:737-43.

24. Boermsa F, Van den Brink W, Deeg DJ, Eefsting JA, Van Tilburg W. Survival in a population-based cohort of dementia patients: predictors and causes of mortality. Int J Geriatr Psychiatry. 1999;14:748-53.

25. Saz P, Launer LJ, Día JL, De-La-Cámara C, Mar$\cos$ G; Lobo A. Mortality and mental disorders in Spanishs elderly population. Int J Geriatr Psychiatry. 1999; 14(12): 1031-8.

26. Dewey ME, Saz P. Dementia, cognitive impairment and mortality in persons aged 65 and over living in the community: a systematic review of the literature. Int J Geriatr Psychiatry. 2001: 16(8): 751-61.

27. Puig X, Gispert R, Puigdefàbregas A, Pérez G, Mompart A, Domènech J. Mortalidad por demencias en Cataluña: un problema de salud emergente. Med Clin (Barc). 2002; 118: 455-9.

28. Corrada M, Brookmeyer R, Kawas C. Sources of variability in prevalence rates of Alzheimer's disease. Int J Epidemiol. 1995;24:1000-1005.

29. Ritchie K, Kildea D. Is senile dementia "agerelated" or "ageing-related"? evidence from metaanalysis of dementia prevalence in the oldest old. Lancet.. 1995;346:931-934.
30. Aronson MK, Ooi WL, Geva DL, Masur D, Blau A, Frishman W. Dementia: age-dependent incidence, prevalence, and mortality in the old old. Arch Intern Med. 1991;151:989-992.

31. Bachman DL, Wolf PA, Linn R, Knoefel JE, Cobb J, Belanger A, D'Agostino RB, et al. Prevalence of dementia and probably senile dementia of the Alzheimer type in the Framingham study. Neurology. 1992;42:115-119.

32. Molsa PK, Marttila RJ, Rinne UK. Long-term survival and predictors of mortality in Alzheimer's disease and multi-infarct dementia. Acta Neurol Scand. 1995;91:159-64.

33. Aevarsson O, Skoog I. Dementai disorders in a birth cohort followed form age 85 to 88 : the influence of mortality, refusal rate, and diagnostic change on prevalence. Int Psychogeriatr. 1997;9:11-23.

34. Helmer C, Damon D, Letenneur L, Fabrigoule C, Barberger-Gateau P, Lafont S, et al. Marital status and risk of Alzheimer's disease: a French population-based cohort study. Neurology. 1999 Dec 10;53(9):1953-8.

35. Turró-Garriga O, Soler-Cors O, Garre-Olmo J, López-Pousa S, Vilalta-Franch j, Monserrat-Villa S. Distribución factorial de la carga en cuidadores de pacientes con enfermedad de Alzheimer. Rev Neurol. 2008 ; 46: 582-588. 